\section{TMSGN: A program for testing trend against a nonrandom alternative}

\author{
RONALD L. RAY, HENRY H. EMURIAN, \\ and RICHARD M. WURSTER \\ Johns Hopkins University, School of Medicine \\ Baltimore, Maryland 21205
}

A simple test of trend in a time series with high asymptotic power is Cox's sign test (Cox \& Stuart, 1955). In this test, values from the middle third of the time series are ignored and values from the last third of the series are compared with values from the first third of the series. More precisely, let $n^{\prime}$ equal $(N+1) / 3$, where $\mathbf{N}$ is the total length of the series. Each value in the first one-third of the series is subtracted from the value in the last one-third of the series, which follows by $2 n^{\prime}$ observations. The number of these differences that are positive in sign is noted. If the number of positive differences is $r$, then

$$
\left(r-n^{\prime} / 2\right) / \sqrt{\left.n^{\prime} / 4\right)}
$$

is approximately distributed as Z. A potential problem with this technique is that under the null hypothesis the series is assumed to be random, with no serial correlation among the individual values. Many behavioral or physiological series are not random but, instead, exhibit substantial serial correlation among their successive values. If the above test is applied to such series, the probability associated with the obtained approximate $Z$ value will be substantially different from that shown in a standard $Z$ table. Goodman (1963) and Sen (1965) have provided modifications to allow for serial correlation among values in the series. These modifications can best be understood by realizing that the foregoing sign test can be reconceptualized as a process of differencing a series of data points separated by $2 n^{\prime}$ intervening data points, setting all positively signed differences equal to one, and setting all negatively signed differences equal to zero. The sum of the series of dichotomous values is $r$, and if there is no serial correlation present, the standard error of the expected sum is $\sqrt{n^{\prime} / 4}$. The Goodman (1963) and Sen (1965) results provide a modified standard error based on the characterization of the series of dichotomous values as an M-dependent series. An M-dependent series is a series of values that exhibits no serial correlation among its member values, which are separated by more than $M$ intervening values. For an M-dependent series, the standard error of $r$ is modified by adding a term that is proportional to the $\mathbf{M}$ autocovariances of the series. The $\mathrm{K}$ th autocovariance is defined by:

$$
\mathrm{R}_{\mathrm{k}}=\sum_{\mathrm{i}=1}^{\mathrm{n}^{\prime}-\mathrm{k}} \mathrm{U}_{\mathrm{i}} \mathrm{U}_{\mathrm{i}+\mathrm{k}} /\left(\mathrm{n}^{\prime}-\mathrm{k}\right)-\mathrm{M}^{2}
$$

where $U_{i}$ is the ith member of the series of dichotomous values, $K$ is the lag of the autocovariance, $M$ is the mean of the series, and $n^{\prime}$ is the length of the transformed series. The modified variance of $U_{1}$ is then

$$
\operatorname{VAR} *\left(U_{i}\right)=R_{0}+2 \sum_{k=1}^{m} R_{k},
$$

and the standard error of $r$ for the modified sign test is

$$
\operatorname{SE}^{*}(\mathrm{r})=\sqrt{\mathrm{n}^{\prime} \mathrm{VAR}^{*}\left(\mathrm{U}_{\mathrm{i}}\right)}
$$

For an M-dependent series, $\left(r-n^{\prime} / 2\right) / S E^{*}(r)$ is approximately distributed as $Z$. The major problems associated with the use of this test are related to the degree of dependency exhibited by the series. The true value of $M$ must be much smaller than $n^{\prime}$. And, a reasonable estimate of the value of $M$ must be obtained. A practical approach is to estimate the autocorrelation function of the series of differenced (but not dichotomized) values. If the autocorrelations are close to zero after a certain lag, then $M$ is set equal to that lag. In order to do this with any confidence, the series of differenced values must include at least $50-100$ values.

The time-series sign test can also be used to test for an " $\mathrm{N}$ of 1 " treatment effect in an $\mathrm{AB}$ design. In this case, the differences used to obtain the transformed values include the first value of Period A subtracted from the first value of Period $B$, the second value of Period A subtracted from the second value of Period B, and so on. In other words, $n^{\prime}$ is set equal to the number of values in either $\mathbf{A}$ or $\mathbf{B}$, whichever was less.

Paradoxically, the time-series sign test cannot be used if a very potent trend or treatment effect is present. If, for example, the data consist of a perfect monotonic trend, all the autocovariances will be equal to the variance and the variance adjustment will result in a variance estimate that always increases with $M$. The test will be most useful in situations in which such tests should be most useful (i.e., in a situation in which there is doubt about the presence of trend or of a treatment effect).

Description. The TMSGN program is available in a PDP-8 OS78 BASIC version, a FORTRAN IV batch version, and a PLI batch version. The BASIC program inputs the data file name, the number of data points, the order of $\mathrm{M}$ dependency, and the differencing constant from the keyboard. The data file is assumed to be stored on a disk as a string of numerical values 
separated by commas. In the FORTRAN IV and PLI versions, the program parameters and data values are input from cards in the same order as in the BASIC program. An object-time format card is also required with the FORTRAN IV version. After reading the data, the programs difference and dichotomize the data values and compute the value of $Z$ appropriate under the hypothesis of zero dependency in the series, the autocovariance for each lag up to $M$, and the value of $Z$ corrected for $\mathbf{M}$ dependency.

Restrictions. The program ignores tied values, and series with more than a few tied values should not be analyzed in this fashion. The decision as to the actual number of tied values that is admissible should be determined by the individual user's decision as to what percent of the data he or she is prepared to ignore. Also, since the significance test requires the use of fairly long series, the series length should probably be equal to at least 100 or more observations.

Availability. A source listing, a user's handout, and sample input can be obtained free of charge from R. L. Ray, Phipps E-2, 600 N. Wolfe Street, Baltimore, Maryland 21205.

\section{REFERENCES}

Cox, D. R., \& Stuart, A. Some quick sign tests for trend in location and dispersion. Biometrica, 1955, 42, 80-95.

Goodman, L. Tests based upon the movements in and the comovements between $\mathrm{M}$-dependent time-series. In C. Christ (Ed.), Measurement in economics. Stanford, Calif: Stanford University Press, 1963.

Sen, P. K. Some non-parametric tests for M-dependent time series. Journal of the American Statistical Association, 1965, $60,134-147$.

(Accepted for publication February 25, 1982.) 